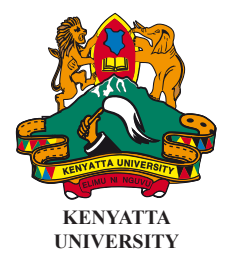

ISSN 1563-1028
CHEMCHEMI

International Journal of Humanities and Social Sciences

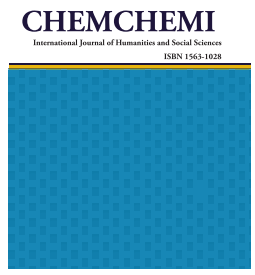

Volume 10 Number 2, 2016

\title{
CONCILIER FORMATION INITIALE ET PRATIQUE D'ENSEIGNEMENT DU FLE EN CONTEXTE KENYAN
}

\author{
Caroline C. A. Oyugi
}

\begin{abstract}
Language facilitates the sharing of one's personal experiences with another. Thus, teaching and learning a language is anchored on interaction. The learner learns to communicate through authentic communication; in the context of a foreign language, the language is therefore both the tool and object of learning. Thematic training for teachers in the field of foreign language is scanty. The usual initial training course offers future instructors theoretical baggage in methodology, linguistics, and some literary areas. In some instances, exposure to cultural studies and emerging practices such as the use of authentic materials in language classes may take place. While the existing models largely target the adolescent public, the observations and practices meant to allow trainees to acquire baseline experience remain insufficient.

The proliferation of tertiary institutions where foreign languages are taught has in turn spurred teacher mobility in response to the new opportunities. This mobility, based on the desire for professional growth, has seen teachers trained for the secondary school level moving on to adult teaching post-secondary establishments. Supposing that each public has specific learning and language needs which correspond to diverse social contexts, a number of questions need addressing: in the context of evolving needs, are instructors aware of their roles? How do they ensure that learning takes place? What mechanisms are put in place to ensure learning that responds not only to administrative demands but also to learners' needs? In order to operate effectively across different academic and linguistic levels, are there methodological techniques that allow differentiated perception of the material at hand and more specifically in our context, facilitate learning of French as a Foreign Language?

This paper is born of a study on the gaps existing between the theoretical expectations of initial training of instructors in the field of French as a foreign language and the reality on the evolving context on the ground. This paper makes certain proposals regarding the recruitment and continuing professional development of French language teachers, all with the intention of enhancing their efficiency in the field.
\end{abstract}

\section{Le Français dans le système scolaire kenyan}

Les traces initiales de l'apprentissage de la langue française au Kenya remontent à 1967, quatre ans après la fin de l'ère coloniale (Chokah, 2012). À cette époque-là, certains cadres fonctionnaires du Ministère du tourisme et de celui des Affaires Étrangères ou à ses ambassades ouvertes à l'étranger suivaient des cours de français dont l'enseignement était assuré par des enseignants expatriés de la France et du Canada.

La langue française a été introduite dans le système scolaire comme matière spéciale en 1964, figurant 
au cursus d'une dizaine d'écoles secondaires gérées par les congrégations religieuses issues des pays francophones. Le choix de ces écoles était plutôt le résultat des décisions propres aux décideurs privés que d'une politique particulière mise en place pour assurer le contact répandu de la langue française au pays.

Au cours des années 70, environ 15 écoles secondaires dans la capitale, Nairobi, assuraient des cours de français pendant quatre des six années que comportait la durée du cycle d'études. Dans les milieux ruraux, un nombre restreint d'écoles ont pareillement engagé des services des enseignants immigrés des pays voisins. Plus spécifiquement, le Rwanda et le Burundi, pays francophones voisins, et l'Ouganda un pays anglophone, qui à cette période, était secoué par la turbulence politique, sont devenus les sources principales des enseignants recrutés à titre privé par les écoles kenyanes pour enseigner certaines matières manquant assez de professeurs, y compris le français. Au cours des années 80, le Ministère de l'Education s'intéressait à la mise en place d'un programme cohérent visant la sensibilisation à l'importance des langues étrangères dans les écoles secondaires et les établissements d'enseignement tertiaires. Durant cette décennie, le nombre d'écoles où l'on pouvait apprendre le français a augmenté d'une vingtaine à plus de cinquante.

En même temps, pour répondre au besoin des professeurs formés, deux écoles normales d'institutrices, à savoir : Siriba Teachers College et Kisii College situées dans des villes de Maseno et Kisii respectivement, étaient ciblées comme établissements de formation de professeurs de la langue française. Après avoir suivi deux ans de formation pédagogique comprenant un trimestre de stage pratique, les étudiants devenaient titulaires du Diploma in Humanities (Diplôme en sciences humaines), voire la certification minimum leur permettant d'exercer leurs fonctions professionnelles dans les écoles secondaires.

Désireux de mettre en œuvre une politique de nationalisation de postes publiques, le Ministère de l'Education a progressivement remplacé des enseignants-expatriés par des citoyens habilités. Le renouvellement a été lent par rapport à l'étatisation de l'enseignement du français car les professeurs formés étaient aussi recherchés par les organismes privés non-scolaires, afin de renforcer leurs fonctions d'intendance. À présent, sauf des établissements scolaires privés n’engagent des services des enseignants non-kenyans et ceci en contrat de durée indéterminée.

Siriba Teachers College et Kisii College ont depuis évolué aux instituts d'enseignement supérieur rattachés aux universités et à nos jours, leurs fonctions de jadis ont été transférées à deux autres institutions, notamment Kagumo College, dans la région centrale du pays et Kibabï College situé à l'Ouest du pays.

\section{Le français au niveau primaire et secondaire}

Dans le cycle primaire, l'apprentissage du français au sens formel est limité à un public très restreint, essentiellement dans les écoles internationales et certains établissements privés dans des grandes villes du pays. Pourtant, le club de français existe dans plusieurs écoles, y compris celles publiques. L'enseignement d'une langue étrangère n'est proposé qu'à partir du cycle secondaire et toujours en tant que matière facultative.

De nos jours, l'apprentissage du français est répandu dans les écoles secondaires nationales et provinciales. La langue est enseignée dans sept des huit régions administratives, absente au nord-est du pays. Néanmoins, parmi les langues étrangères enseignées au niveau secondaire, c'est le français qui reste la langue étrangère la plus sollicitée. 
L'entrée du français dans le programme d'un lycée dépend largement de trois facteurs : premièrement et portant plus de poids c'est la volonté de la communauté parrainant l'école. Cet élément est important puisque les souhaits des communautés bienfaiteurs sont pris en compte par le gouvernement central, veillant sur le système scolaire du pays.

En deuxième lieu se trouve la volonté du proviseur de l'école d'avoir une présence vive de la langue dans son établissement. Selon l'opinion anecdotique, il paraît que le sort d'une matière dite facultative, voire sa popularité dans l'école ou même la suppression de l'emploi du temps dépend considérablement de la bienveillance du directeur de l'école. Le troisième facteur qu'il faut prendre en compte serait la disponibilité d'un enseignant de manière régulière, favorable à l'établissement ainsi que le maintien de la langue française dans une école.

Le français est actuellement enseigné dans environ 350 écoles secondaires ${ }^{2}$ à un public d'approximativement 29000 élèves dont un chiffre d'environ $9 \%$ des candidats se présentent annuellement à l'examen KCSE. Ceci est encore bas par rapport à la projection du Ministère d'éducation nationale qui prévoit atteindre un seuil de $15 \%$. Le graphique suivant nous montre la répartition des écoles où le français est enseigné selon les régions administratives:

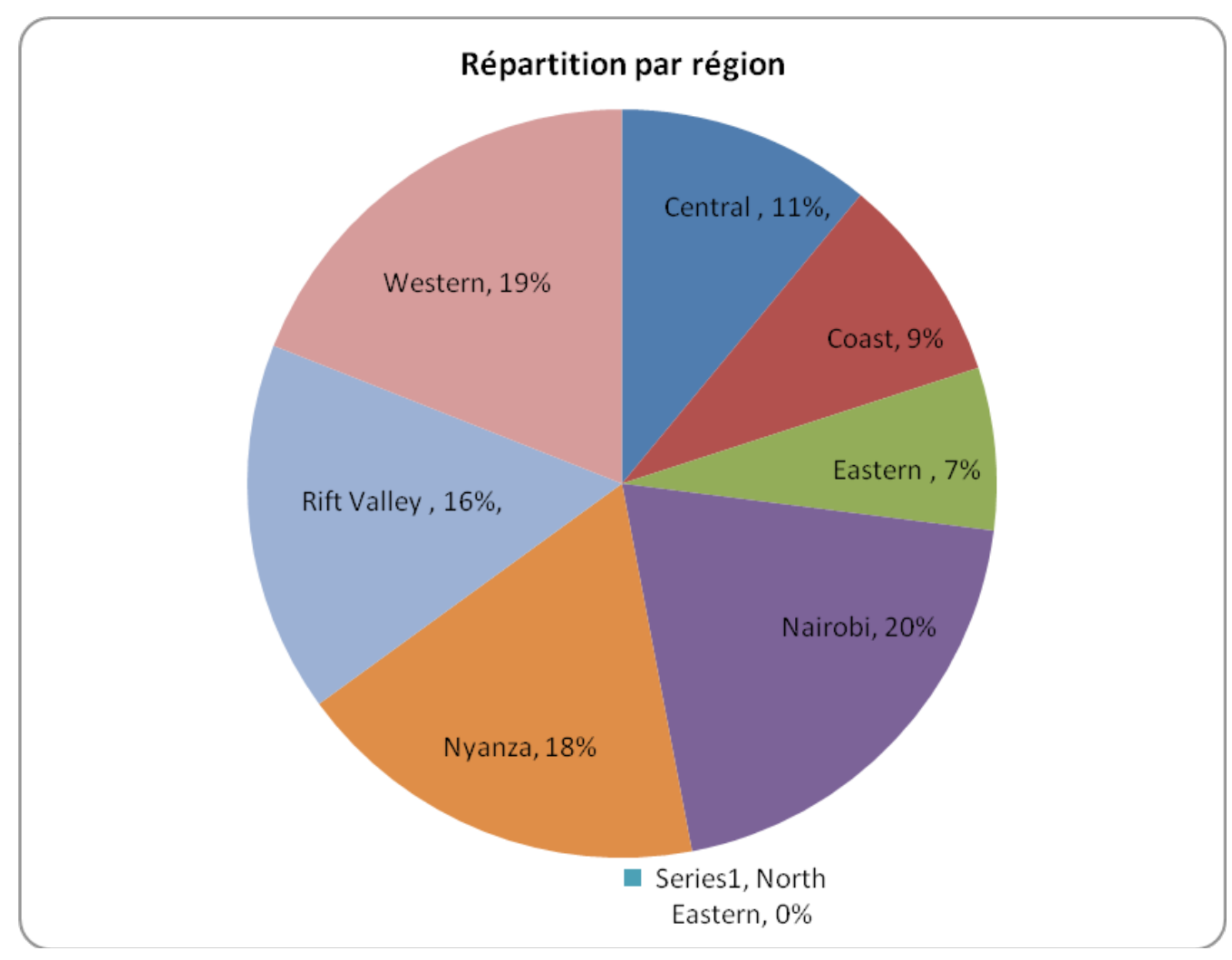

\section{L'enseignement du français au niveau universitaire *}

Au niveau universitaire au Kenya, la langue française est présente dans les six universités publiques et sept universités privées. L’apprentissage du français à ce niveau est de façon générale facultatif. Toutefois, certaines universités, en exerçant les droits que leur confèrent leurs chartes respectives ont mis en place certains cours exigeant la connaissance complémentaire d'une langue étrangère (selon KUCCPS, 2015). 
Pour illustration, United States International University (USIU), un établissement privé, exige que tous les étudiants inscrits au cours de licence suivent également six mois de cours en langue étrangère. À Moï University, par contre, la langue française est obligatoire pour tous étudiants inscrits en licence au département du tourisme. Les étudiants inscrits à Kenyatta University, eux, ont l'opportunité de suivre les cours de langue française en complémentarité avec les sujets principaux, dits majeurs. L'Institut de diplomatie et d'études internationales situé à l'Université de Nairobi exige aussi des cours de langue étrangères aux inscrits.

Dans les universités privées, les cours de langues étrangères sont disponibles pendant certains semestres et sont recommandés aux étudiants plutôt à titre optionnel. Par contre, United States International University exige un nombre spécifique d'heures à valider en cours de langue avant que les étudiants soient admissibles pour le stage professionnel.

Pourtant, afin de donner une représentation plus globale des positions diverses prises par les universités par rapport à la langue française ainsi qu'illustrer l'étendue des programmes d'études disponibles en français au niveau universitaire, notre étude propose ce tableau 1 ci-après: 
Tableau 1: Programmes de français au niveau universitaire

\begin{tabular}{|c|c|}
\hline Université & Statut du français \\
\hline $\begin{array}{l}\text { Catholic University of East } \\
\text { Africa }-\mathrm{Pv}\end{array}$ & Cours de langue: niveau débutant - facultatifs \\
\hline Daystar University - Pv & $\begin{array}{l}\text { i. Licence en lettres : option études en français } \\
\text { ii. Licence en pédagogie } \\
\text { iii. Cours de langue : niveau débutant - facultatifs }\end{array}$ \\
\hline Egerton University - P & Cours de langue : niveau débutant - facultatifs \\
\hline Kenya Methodist University & $\begin{array}{l}\text { Cours de langue, littérature, traduction et } \\
\text { communication }\end{array}$ \\
\hline Kenyatta University - P & $\begin{array}{l}\text { i. Licence en lettres : option études en français } \\
\text { ii. Licence en didactique : option français et une } \\
\text { deuxième matière } \\
\text { iii. Maîtrise en français: option : } \\
\text { a.) Français langue étrangère } \\
\text { b.) Linguistique }\end{array}$ \\
\hline Maasai Mara University - P & $\begin{array}{l}\text { Cours de langue française générale ; français de } \\
\text { spécialité, option tourisme. }\end{array}$ \\
\hline Maseno University - P & $\begin{array}{l}\text { i. Licence en didactique : option français } \\
\text { ii. Français sur objectifs spécifiques (FOS): Tourisme } \\
\text { iii. Maîtrise en didactique } \\
\text { iv. Unité doctorale }\end{array}$ \\
\hline $\begin{array}{l}\text { Masinde Muliro University of } \\
\text { Science and Technology - P }\end{array}$ & $\begin{array}{l}\text { i. Licence en didactique : option français et une } \\
\text { deuxième matière } \\
\text { ii. Initiation à la langue française - facultative }\end{array}$ \\
\hline Moï University - P & $\begin{array}{l}\text { i. Cours de français élémentaire : obligatoires en } \\
\text { première année } \\
\text { ii. Français de Spécialité: option tourisme }\end{array}$ \\
\hline Mount Kenya University $-\mathrm{Pv}$ & Cours de langue générale \\
\hline Strathmore University - Pv & $\begin{array}{l}\text { Cours de langue: facultatifs - niveaux débutant et } \\
\text { intermédiaire }\end{array}$ \\
\hline $\begin{array}{l}\text { United States International } \\
\text { University }-\mathrm{Pv}\end{array}$ & $\begin{array}{l}\text { Cours de langue élémentaire: obligatoires en premier } \\
\text { cycle }\end{array}$ \\
\hline $\begin{array}{l}\text { University of East Africa: } \\
\text { Baraton - Pv }\end{array}$ & Etudes de langue: facultatives aux débutants \\
\hline University of Nairobi - P & $\begin{array}{l}\text { i. Licence en lettres : option - études en français } \\
\text { ii. FOS: options études internationales, tourisme et } \\
\text { journalisme } \\
\text { iii. Cours de français élémentaire : facultatifs - niveau } \\
\text { débutant }\end{array}$ \\
\hline
\end{tabular}

À partir des renseignements que fournit le tableau, nous notons que l'éventail de cours mis en place s'étend de ceux destinés aux vrais débutants à une école doctorale. Les dispositifs ciblant la formation de bacheliers ainsi que les personnes en domaines de spécialisation comme le tourisme, le journalisme et l'hôtellerie s'imposent dans la plupart des universités, privées comme publiques.

Selon le cite officiel de l'Alliance Francaise, le réseau de l'Alliance Française au Kenya comprend le siège à Nairobi, et des agences dans les villes principales de Mombasa situé dans la région côtière, Kisumu, à Nyanza et Eldoret situé à Rift Valley. Il y a deux ans, L'Alliance Française de Nairobi a généré un 
établissement secondaire à Buru-Buru, un quartier moyen classe parmi les plus peuplés de la capitale. Cette démarche vers la décentralisation de cours a été prise en vue de la longue liste d'attente pour certains modules y compris ceux comportant le français intermédiaire et le français commercial. L’expansion de l'Alliance Française renforce son rôle opérateur clé de diffusion de la langue parmi la population kenyane ; ses enseignants suivant plutôt des formations très particulières à son contexte.

La population atteinte par L'Alliance Française s'étend des étudiants qui suivent des formations diplômantes aux professeurs de FLE suivant des stages intensifs ou voulant simplement profiter des ressources mis à leur disposition dans le centre de documentation pédagogique. La salle polyvalente et l'auditorium offrent aussi la possibilité d'interaction avec la langue française au cours des évènements informels.

Étudier dans les collèges spécialisés est une autre option se présentant aux étudiants kenyans. Afin d'attirer des apprenants, ainsi qu'aiguiser leur avantage concurrentiel, ces collèges, compris dans le sens des établissements d'enseignement du niveau postsecondaires, sont parfois obligés par les demandes du marché d'intégrer le français dans leurs programmes d'études.

Pour la plupart privées, les écoles polytechniques et instituts techniques proposent un enseignement spécialisé orienté vers des domaines distinctifs y compris les métiers du tourisme, l'industrie hospitalière et le journalisme. Bien que le niveau de langue enseigné dans les établissements reste essentiellement de l'élémentaire à l'intermédiaire, (Chokah, 2012) l'inclusion du français dans les programmes des collèges aurait deux buts : d'une part, offrir aux étudiants un forfait plus attrayant et d'autre part, ils deviendraient des terrains de choix du recrutement par les employeurs.

\section{Objectifs du français au niveau universitaire}

D'après le Kenya Institute of Curriculum Development (KICD), l'Institut chargé de l'édition des ouvrages scolaires, l'enseignement du français dans les écoles secondaires au Kenya répond à quatre objectifs primordiaux, à savoir:

i. pourvoir les apprenants dans les écoles secondaires de compétences linguistiques de base afin qu'ils puissent communiquer en français là où ils en auront besoin;

ii. accorder aux apprenants l'accès aux matériels oraux et écrits en français;

iii. faciliter les études supérieures dans des établissements francophones;

iv. promouvoir la paix mondiale à travers la compréhension et l'appréciation des cultures francophones, par une perception positive des étrangers et leurs cultures

Une analyse générale des objectifs énumérés en révèle la pensée sous-tendant. Tout d'abord, que l'apprentissage de français dans le contexte kenyan vise la communication appropriée en diverses circonstances. L'apprentissage favorisera également l'ouverture de l'esprit de l'élève aux opportunités du progrès scolaire au-delà de l'environnement local familier.

Les universités visent à renforcer ces mêmes objectifs mais en mettant plus d'accent sur les opportunités du marché du travail. Loin de ne donner que des cours de sensibilisation à la langue française, la liste d'études offertes montre que de façon générale, ces établissements visent à attirer des étudiants qui s’intéressent 
à se spécialiser dans une diversité de domaines, tels que le tourisme et l'hôtellerie, la diplomatie et le journalisme.

En outre, enseigner le français dans les établissements d'études supérieures au Kenya ambitionne à influer sur le domaine affectif de l'étudiant, c'est-à-dire l'aider à parvenir à un certain niveau d'entendement et d'estimation de l'autre, originaire de la culture francophone. Afin d'assurer que le français soit enseigné dans des meilleures conditions possibles, les universités sont toujours à la recherche des meilleurs enseignants compétents qui peuvent adapter le matériel pédagogique approuvé par le KICD pour les niveaux inférieurs, pour satisfaire les besoins du public adulte.

$\mathrm{Au}$ niveau secondaire, où l'on trouve le grand pourcentage des apprenants du français, les matériaux pédagogiques fondamentaux disponibles à un prix abordable comprennent les suivants:

i. le manuel ou ouvrage de références destiné à l'usage de l'élève. À part des explications et illustrations pertinentes, le manuel sert aussi de source des exercices d'entraînement sur la variété des sujets.

ii. un guide pédagogique au professeur présentant des modèles des démarches possibles dans l'enseignement ainsi que des réponses aux exercices proposés aux élèves.

iii. une cassette audio illustrant des parties sonores de chaque unité.

iv. une cassette d'entraînement des méthodes accompagnées par des bandes sonores illustrant certaines parties des cours.

Quant au matériel accessoire, les méthodes auxiliaires surtout celles en provenance des maisons d'éditions françaises, sont aussi disponibles comme ouvrages de référence aux professeurs. Certaines écoles plus douées ont investi en téléviseurs, les disques et lecteurs numériques ainsi que des ordinateurs mis à la disponibilité des apprenants. Cependant, les médiathèques au sein de l'Alliance Française régionales fournissent aux intéressés des ressources additionnels leur aidant à améliorer leur connaissance de la langue française.

\section{Profil des professeurs de français}

Selon l'annuaire des professeurs de français 2012-2014, le corps professoral compte approximativement 440 professeurs dans les écoles secondaires publiques et à peu près cinquante-cinq dans les universités. Dans ces dernières, le recrutement se fait sur la base des concours internes décentralisés. Ceci signifie que chaque université peut embaucher des enseignants selon des critères qui lui sont propres. Par conséquent, on trouve que pour les cours élémentaires, les universités recourent aux professeurs expérimentés des écoles qui possèdent l'habileté minimum leur permettant de fonctionner dans un établissement d'enseignement supérieur. La plupart sont des professeurs recrutés à mi-temps ou à titre vacataire. C'est pour cette raison que, établir le nombre exact de professeur de français dans des différentes catégories d'établissement reste une tâche compliquée.

Quant aux qualifications professionnelles, 12,1\% de la fraternité professorale détient d'une maîtrise en français. Les professeurs ayant ce diplôme trouvent des opportunités d'avancement professionnel en tant que maitres de conférences dans les collèges spécialisés. Selon les données disponibles jusqu’à fin janvier 2014 et sur la base du diplôme le plus récemment reçu, la communauté d'enseignants de français au Kenya est ainsi repartie: 


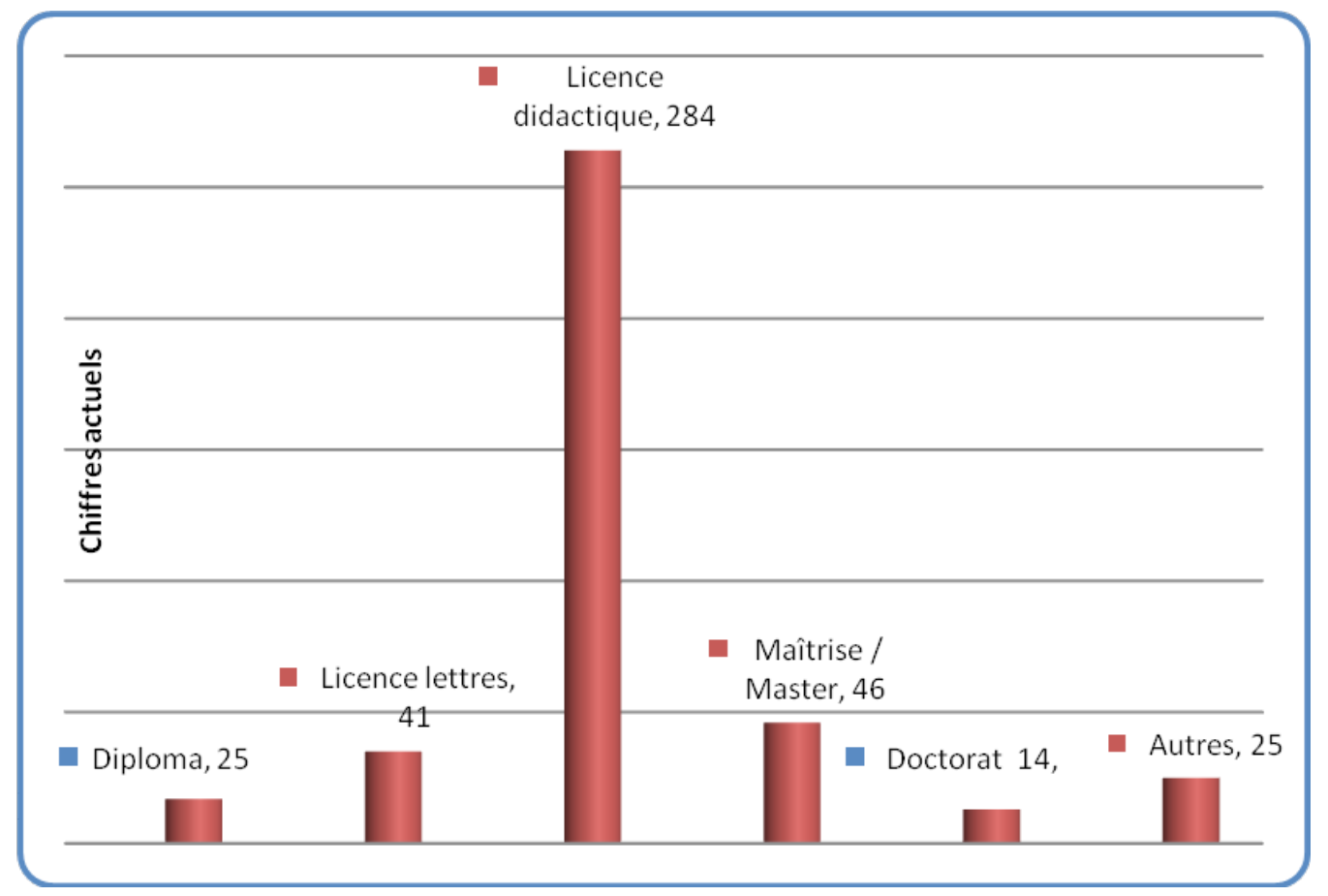

\section{Le maître des conférences que fait-il exactement ?}

Partant de la prémisse que la classe de langue représente d'une part, un lieu d'échanges sociaux et de l'autre part, une situation formelle donnant lieu à l'exposition des faits langagiers se caractérisant par des comportements types générés par le statut des participants, notre présentation se porte aux paramètres entrant en jeu, tant qu'ils sont définis par Cicurel (2011), par rapport à l'agir professoral auprès des étudiants.

L'enseignement au niveau universitaire renvoie à une relation entre des individus dont les rôles généraux nous sont délimités de façon distincte (Prost, 1985). L'étudiant dans notre contexte est celui qui, pour la plupart de temps, est en posture de réceptivité par rapport aux fonctions du professeur. En effet, celui qui donne cours est le professeur ; ce posture le chargeant d'une potentialité à être 'le' supposer savoir et, de ce fait investi d'un rôle particulier (Anzieu, 2003).

Le professeur dans l'espace de sa classe occupe tout d'abord un rôle lui est confié par l'université dont il serait agent. Ainsi, il est chargé de la fonction de transmettre du savoir et d'en permettre la construction et l'appropriation comme nous le dit Cifali (2005). Toutefois, il paraît que le processus de transmission de savoirs constitue en lui un enchaînement d'actions annexées discernables en pratique. La série de faits propre à chaque contexte d'enseignement serait influée par des attributs circonstanciels réunissant à la fois les prédispositions méthodologiques et l'image que porte le professeur de ses tâches. Pour donner une forme de structure à notre répertoire de rôles principaux, nous adoptons la triple fonction constituée des axes interdépendants de l'enseignement, à savoir : informer, animer et évaluer (Dabène, 1984).

Le professeur de langue étrangère est vu dans un premier temps, comme dépositaire d'une autre langue qu'il actualise dans l'acte d'enseignement. À une façon propre à lui, le professeur de langue étrangère partage l'information sur la nature et le fonctionnement de ses structures de façon séquentielle aux étudiants (Baï etto, 1980). Cette fonction est remarquée pendant les moments que l'enseignant prend pour reconstruire 
les savoirs afin qu'ils soient abordables par les étudiants selon leur niveau (Anzieu, 2003).

Cependant, la fonction d'expliquer ainsi que ;

[...] faire comprendre les vocables étrangers n'est qu'une partie de la tâche du professeur [...]. Le but à atteindre, c'est que ces vocables deviennent tellement familiers aux élèves quils en disposent à volonté, quils soient assimilés [...] (Simonnot \& Schweitzer, 1921 p. 170).

Ainsi, la fonction de transmission de savoirs se fait d'une manière progressive pour que les étudiants ne deviennent pas dépendants d'information mais visent la maîtrise de la matière dont les démarches suivies progressent de l'apprentissage par imitation servile à la phase de la reconstruction et l'intégration aux savoirs précédentes (Prost, 1985).

De la même façon, l'exposition s'aperçoit tandis que le professeur explicite le fonctionnement de certains structures tout en leur mettant en parallèle aux langues de références des élèves. D'après Beacco et al., (2007 p. 28), l'exposition à la langue cible s'effectue au moyen d'échantillons, c'est-à-dire " de fragments limités à la langue cible [...] qui sont représentatifs de celle-ci» construisant donc un objet - langue pour l'enseignement.

Une autre attente clé du professeur de français langue étrangère est qu'il soit capable d'éveilleur d'intérêt à la langue. Ainsi, être professeur implique assumer en même temps, la présentation de savoirs et le suivi de leur appropriation (Meirieu, 2005). Il s'ensuit que c'est le professeur qui accorde l'accès aux savoirs dont le partage se fait de façon cadencée. Ainsi, il éveille chez ses étudiants l'intérêt dans la langue qu'on apprend (Prost, 1985).

La curiosité née dans l'esprit des apprenants leur donne, à son tour, le goût de l'approprier. Pour cette raison, le professeur tient encore la responsabilité de préserver la petite flamme existant chez l'étudiant (Steiner et Ladjali, 2003) afin de soutenir le désir pour l'objet qui se montre à travers des questions posés par les étudiants. A ce niveau d'apprentissage, l'approche méthodologique diffère car " ce n'est pas à celui qui détient le savoir de poser des questions à celui qui ne sait pas [...]» (Mathis, 1997 p. 71).

La phase de curiosité active se révèle fondamentale puisque qu'elle sert à rendre les étudiants demandeurs de la parole du professeur; celui qui apprend doit satisfaire sa curiosité par des répliques reçues aux questions qu'il pose à sa propre volonté. Il pourrait bien sortir qu'en se montrant accueillant et compréhensif de la logique, le raisonnement et les problèmes des étudiants, le professeur se trouve en rôle de médiateur, fournissant le rôle du lien entre les étudiants et les savoirs (Romano et Salzer, 1987).

La mise en œuvre du rôle prescrit qu'il conçoive les instruments et les démarches permettant à l'étudiant d'évoluer dans le but de franchir le stade du récepteur passive(Bronckart, 1977), qui doit quitter ce qui le handicape, et consente à apprendre et par contre, accéder au plaisir qu'éprouve le sujet ayant approprié la langue de l'autre (Cifali, 2005). Qui est plus, le rôle d'éveil à la langue s'étend de susciter le désir chez l'étudiant par rapport aux mécanismes codifiant la langue au maintien de contact de façon continue avec le sujet. Ainsi, en faisant travailler les étudiants, le professeur veille à valoriser les efforts constatés vers à la prise d'initiative et favorisant la confrontation avec la langue au plan personnel.

En fonction d'animateur, il convient de préciser que de façon générale, au début de la leçon, c'est principalement le professeur-animateur qui est à la fois au courant du contenu de l'étude et est aussi en 
conscience de la progression souhaitée dans chaque séance en classe. En ce rôle, le professeur organise ou planifie les séquences du cours dans la visée d'assurer la cohérence des savoirs transmis aux élèves.

Les étapes gestionnaires elles-mêmes consisteraient à exposer, non seulement les tournures particulières aux aspects phonétiques de la langue mais également les différents consignes facilitant les interactions entre le professeur-animateur et les étudiants comme entre les étudiants eux-mêmes. Dans la perspective que la classe de langue ait le caractère d'un réseau tous circuits, il retombe au professeur à trouver les mécanismes l'aidant à tisser et à maintenir une ambiance propice à l'interaction équilibre.

Ne la progression du cours étant conçue dans une logique provenant de la maîtrise des séquences et celle des connaissances, la fonction gestionnaire d'enseignement revendique que le professeur s'apprête à gérer l'imprévu (Puren, 1999). Vue contre les structures mises en place au niveau secondaire, le fortuit, à l'université, devient la norme car il se présenterait en certaines formes telles que l'ajustement de parcours pour prendre compte d'hétérogénéité des étudiants ou gérer les perturbations surgissant au cours de l'apprentissage. En ce sens, l'apprentissage - animation serait d'une tâche qui s'agit moins d'opportunité que de stratégie (Baïetto, 1980).

Pour cadrer encore les limites de notre exposé, la fonction du guidage décrit le principe général selon lequel l'on trace "le chemin qui convient, y compris dans ses détails [...], d'amener progressivement chaque [étudiant] au but à atteindre en matière d'acquisition de savoirs" (Bru, 2006 p. 46). Le guidage comprendrait donc, l'expérimentation de médiation organisée entre l'objet d'apprentissage et l'apprenant (Cuq et Gruca, 2003). Dans le contexte universitaire au sein de la relation d'appropriation linguistique, l'étudiant serait le bénéficiaire des orientations du professeur, lui-même ici la partie guidant qui agit pour lui montrer la voie.

Quant aux mécanismes de l'accompagnement, le professeur accompagne l'étudiant dans son apprentissage dans le sens qu' il s'intéresse à l'aider à maintenir le contact avec la langue cible, c'est-à-dire, à " [... ] apprécier ses fonctionnements, goûter ces incertitudes ainsi que éprouver ses excentricités.» (Beacco et al, 2007 p. 17). En outre, le professeur étaiera l'étudiant dès qu'il ordonnance ses interrogations afin que le sujet abordé détienne du sens pour lui. Autrement dit, le professeur- accompagnateur " aide l'étudiant à identifier la norme et s'en approprier au bénéfice de sa socialisation " (Blanchet, 1998 p. 67). Nous pouvons constater par la suite que l'accompagnement vise, d'une part, à aider l'étudiant à agencer ses observations et de l'autre part, à confronter sa perception intuitive des fonctionnements de la langue cible.

En fonction d'évaluateur le professeur occupe une position qui l'engage à se prononcer sur l'acceptabilité des productions langagières; rôle "qui le contraint à donner son appréciation après un grand nombre de productions venant des apprenants. " (Dabène et al, 1990 p. 28-29). Autrement dit, c'est le professeur qui détient le dernier mot par rapport à l'acceptabilité des réponses et à ce qu'il faut retenir. À travers le discours didactique, la valorisation des productions de l'étudiant montre le degré de la conformité de la production avec la norme. Cependant, nous notons qu'en situation de classe, le professeur ne peut appréhender que des performances des étudiants, à l'égard des tâches et à des objectifs spécifiques.

Sur la base de ses constats, il peut parvenir à formuler des hypothèses sur les niveaux de performance ultérieurs que les apprenants pourraient atteindre. Restant dans cette optique, connaître les capacités d'apprentissages des élèves ne constitue pas l'évaluation d'un état, mais essentiellement l'aptitude pour définir les étapes nouvelles qu'un étudiant est susceptible de franchir. Nous résumons la réalité hybride du vécu du maitre des conférences en forme du diagramme schématique. 
Figure 3: Fonctions et rôles du professeur

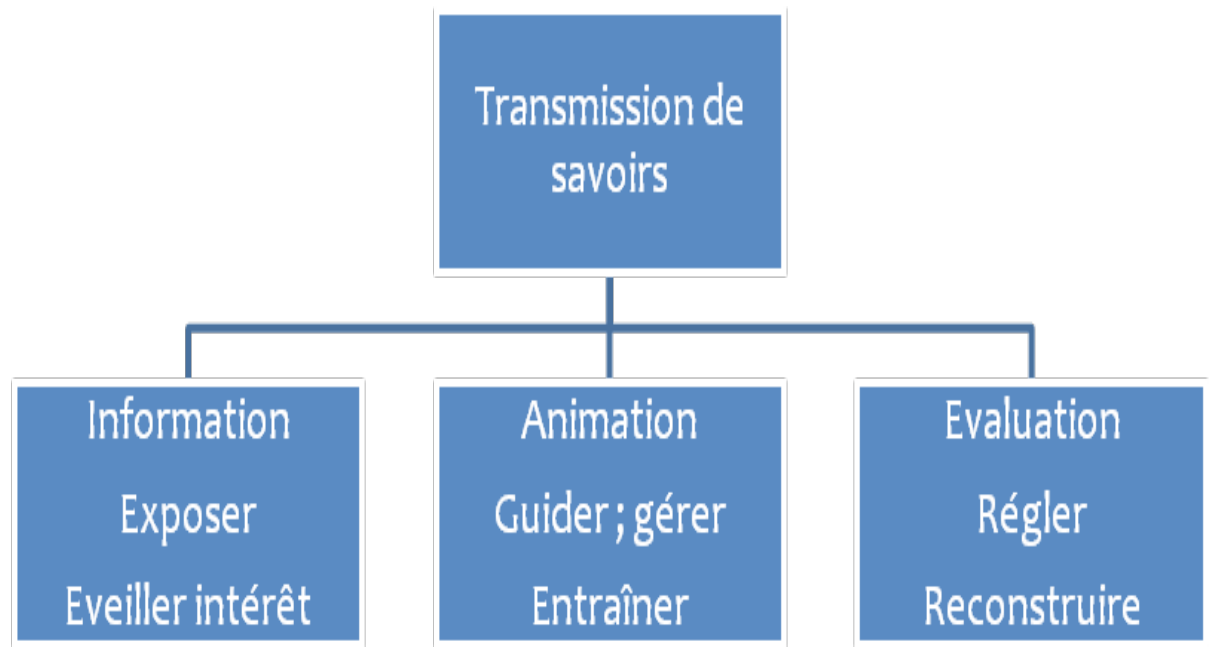

Par notre synthèse, il paraît que se demander qui est maître des conférences, c'est effectuer une sorte d'auto-évaluation. C'est-à-dire, "au-delà d'une analyse de son statut et de son rôle dans l'institution, faire un bilan" tout d'abord de sa personne ; voire, "de ses connaissances, de ses expériences, des formations reçues et des possibilités d'autoformation et de recyclage [...]» (Moirand, 1982 p. 47).

Le processus comprend en même temps une interrogation centré sur l'imagé qu'il a des apprenants et de la langue quil enseigne, sur la représentation qu'il se fait de la communication et de la manière dont on apprend à communiquer, s'est réfléchir enfin sur ses habitudes d'enseignement, ses stratégies d'enseignement, ses capacités à écouter et à observer les apprenants, toujours d'après Moirand (1982 p. 24).

\section{Conflits qu'éprouve le professeur du français langue étrangère}

Ayant établi que pour la plupart les maîtres de conférences, que nous avons observés au travail est spécifiquement formé pour exercer son métier dans un contexte secondaire et public, il lui revient souvent de représenter une langue étrangère dans un univers aussi étranger. Le contexte dans lequel il fonctionne fait de lui maître du langage en usage. Mais l'enseignant, en dépit d'où qu'il se trouve, sert en quelque sorte d'ambassadeur légitime d'un idiome qu'il enseigne et qu'il est supposé connaître mieux que son public (Dabène et al, 1990).

Le professeur est perçu comme celui pour qui le don naturel et la formation trouve leur conjonction dans la communication ; qu'il est habile à se situer par rapport à son public et également trouve le plaisir à communiquer (Sorzana, 2004). Pourtant, il y a lieu de croire que construire une nouvelle situation d'apprentissage oblige le professeur à réinterroger ses propres savoirs et pratiques (Meirieu, 2005). Le contexte de l'enseignant non natif qui avait antérieurement vécu en lui-même l'expérience de l'apprentissage de la langue étrangère qu'il enseigne, risque d'engendrer en lui non seulement "une conscience plus nette des obstacles à franchir ", mais également " un sentiment d'insécurité linguistique qui le rend particulièrement soucieux du respect d'une norme qu'il n'ose transgresser.» (Dabène et al.1990 p. 13)

Les soucis du professeur seraient la source d'angoisses dites professionnelles voire, celles provoquées «par la situation, l'institution, par le rôle instaurée, par les idéalités qui les traversent, par la maitrise supposée, par le héros qu'il devrait être, par le savoir qu'il devrait représenter [...], de façon générale, "l'angoisse qui salimente de n'importe quoi » ayant un rapport avec la situation du travail (Cifali, 2005 p. 94). 
Cependant, l'enseignant est contraint à faire face aux épreuves qui ne seraient pas discernables de façon explicite. En empruntant les propos de Kant (1966 p. 26), "celui qui a tâche de l'éduquer est aussi un homme, affecté par la grossièreté da sa nature, et il doit produire chez l'autre ce dont il a lui-même besoin [...]. "

\section{Conclusion}

L'enseignement et l'apprentissage de la langue française au Kenya suivra la croissance vécue dans le secteur général d'éducation tertiaire. Afin d'attirer et retenir des effectifs importants d'étudiants qui suivront des cursus variés trouvant leur lien dans la langue étrangère, les établissements auront toujours besoin des enseignants non seulement expérimentés mais aussi compétents pour exercer la profession au niveau supérieur. Ceci demande de façon implicite que certaines démarches soient mises en place pour ne pas dégrader les acquis par rapport à l'apprentissage du français.

D'abord, il importe aux enseignants eux-mêmes de prendre l'initiative de renforcer leurs capacités langagières ainsi que leurs habiletés méthodologiques pour pouvoir communiquer efficacement avec le public qu'on leur présente. La mise à jour de ses connaissances linguistiques et didactiques ainsi que les facteurs qui influent sur l'évolution des approches pédagogiques reste une obligation. Actuellement, il ne suffit plus seulement de maîtriser la langue ; il faut aussi savoir comment la partager efficacement, tout en respectant les besoins et exigences du public spécifique.

En outre, on pourrait aussi profiter des ressources déjà existantes dans une vingtaine de lieux différents au pays. On peut citer ici les Centre des ressources Kenyans de français qui offrent un réseau bien équipé où l'on peut exploiter une gamme de matériel visuel, sonore et technique. Mis en place sur un plan régional, ces centres donnent l'occasion de formation des réseaux professionnels en faveur de l'apprentissage et la diffusion de la langue française.

La transmission de savoirs en langue étrangère impose que l'enseignant possède une multiplicité d'habiletés et de compétences linguistiques, techniques et même socioculturelles. Afin d'être efficace en classe, ces capacités professionnelles doivent jouer de façon complémentaire. On ne n’y arrivera pas sans renforcement régulière surtout quand on prend en compte les nombreuses évolutions constaté dans le domaine du FLE. Il est demande de l'enseignant d'être capable d'animer ses cours de manière favorisant l'appropriation de la langue, informer ses élèves selon ses connaissances et propres expériences et aussi faire une estimation juste toute en restant en conformité avec des principes directeurs issus soit du KICD soit de l'établissement d'enseignement supérieur.

Au niveau tertiaire, les demandes des apprentissages varient selon l'institution et, parfois, les exigences du marché. Ici encore, l'enseignant doit savoir comment s'adapter au contexte, de public adulte, dans lequel il se trouve. Pour ceux qui donnent des cours dans les établissements secondaires et aussi au niveau tertiaire, savoir comment et quand modifier son instruction est une compétence à découvrir.

Des formations de réadaptations, des stages thématiques intensifs, l'exploitation efficient des ressources mis en commun et aussi la participation aux activités extra-professionnelles, qui promeuvent la maitrise du français seraient des débouchés vers l’amélioration de sa pratique professionnelle.

Enfin, les professeurs de français sont également incités à profiter des formations en ligne et à distance, tels que la Professionnalisation en français langue étrangère (ProFLE) qu'on vient de lancer au pays et 
aussi le Diplôme en didactique du français pour objectifs spécifiques (DDIFOS) qui cible les pratiquants en français de spécialité (FS) et le français sur objectifs spécifiques (FOS). Ces deux parcours existent en réponse aux tendances globales et seraient bénéfiques à assurer une meilleure formation pour les professeurs de français dans le contexte tertiaire. Le ProFLE en particulier est pertinent en contexte kenyan où l'on trouve un certain pourcentage d'enseignants en fonction sans avoir bénéficié d'une formation initiale en pédagogie de langues étrangères. Le DDIFOS est plutôt adapte aux besoins professionnels des enseignants au niveau supérieur dans lequel ils doivent non seulement donner cours mais aussi concevoir les programmes spécifiquement adaptés à la demande et les objectifs de l'établissement, tout en prenant en compte d'autres facteurs comme : le degré de professionnalisation des apprenants, le niveau de départ en français, la durée de la formation, l'équipement disponible ainsi que le budget alloué à la formation.

Offerts principalement en ligne, elles comprennent la disposition d'accéder à la fois à un nombre d'enseignants qui travailleront à un rythme adapté aux horaires personnels et ceux qui désirent profiter d'une formation continue à distance.

\section{Références}

Aumont, Bernadette \& Mesnier, Pierre-Marie (1992): L'acte d'apprendre, Paris, Presses universitaires de France.

Baieto, Marie - Claude (1980). Le désir d'enseigner, Paris, Éditions ESF.

Beacco, Jean - Claude et al., (2007). L'Approche par compétence des l'enseignement des

langues: enseigner à partir du Cadre européen commun de référence pour les langues, aris, Didier. (2000a). Les dimensions culturelles des enseignements

de langue, Paris, Hachette Livre.

Blanchard - Laville, Claudine, (2001). Les enseignants entre plaisir et souffrance.

Blanchet, P. (1998). Introduction à la complexité de l'enseignement du français langue étrangère, Peeters.

Bouacha, A. Abdelmadjid (dir.) (1978). La pédagogie du français langue étrangère : orientations théoriques, pratiques dans la classe, Paris, Hachette.

Bru M., (2006). Les méthodes en pédagogie, Paris, PUF.

Carlo, Romano \& SALZER, Jacques (1987). Enseigner, c'est aussi savoir communiquer, Paris, les Éditions d'Organisation.

Chokah, Milcah M. (2012). L'Enseignement du Français au Kenya, Nairobi, The Jomo Kenyatta Foundation.

Cicurel, Francine (2011). Parole sur parole ou le métalangage dans la classe de langue, Paris, Clé International. Cifali, Mireille (2005). Le lien éducatif: Contre-jour psychanalytique, Paris, Presses

Universitaires de France.

\& MOLL, Jeanne (1985). Pédagogie et psychanalyse, Paris, BORDAS.

Cordié, Anny (1998). Malaise chez l'enseignant: l'éducation confrontée à la psychanalyse, Editions du Seuil.

Dabène, Louis; CICUREL, Francine et al., (1990). Variations et rituels en classe de langue, Paris, CredifHatier.

Develay, Michel (1992). De L'apprentissage à l'enseignement, Paris, ESF éditeur.

Ganthier, Hélène (1968). L’Enseignement d'une langue étrangère, Paris, Presses universitaires de France.

Gilly, Michel (1980). Qu'est-ce qu'apprendre? Paris, Presses universitaires de France.

Girard, Denis (1995). Enseigner les langues: méthodes et pratiques, Paris, Bordas. 
Guillotte, Alain (1990). Le professeur stratège, Paris, Les Éditions d'Organisation.

Leperlier, Guy (1992). La communication pédagogique : des techniques d'expression au développement personnel, Toulouse, Editions Privat.

Martinez, Pierre (1996). La didactique des langues étrangères, Presses universitaires de France.

Mathis, Geneviève (1997). Professeur de Français : Les clés d'un savoir-faire, Paris, Nathan pédagogie.

Meirieu, Philippe (2005). Lettre à un jeune professeur, ESF éditeur.

Moirand, Sophie et al., (1990). Enseigner à communiquer en langue étrangère, Paris, Hachette.

Perrenoud, Philippe (1996). Enseigner : agir dans l'urgence, décider dans l'incertitude : savoirs et compétences dans un métier complexe, Paris, ESF éditeur.

Porchier, Louis (2004). L'Enseignement des Langues Étrangères, Paris, Hachette éducation.

(1995). Le français langue étrangère : émergence et enseignement d'une discipline, Paris, CNDP, Hachette éducation.

Porquier, Rémy \& PY, Bernard (2004). Apprentissage d'une langue étrangère : contextes et discours, Paris, Didier.

Prost, Antoine (1985). Éloges des pédagogues, Paris, Seuil.

Romano, Carlo \& SALZER, Jacques (1987). Enseigner c'est aussi savoir communiquer, Paris, Éditions d'Organisation.

Sorzana, C. (2004): La prise de parole en public, Paris, Victoires Éditions.

Steiner, George (2003a): Maîtres et disciples, Paris, Gallimard.

\& Ladjali, Cécile (2003b): Éloge de la transmission : Le maitre et l'élève, Paris, Albin Michel.

\section{About the Author}

Dr. Caroline C. A. Oyugi is faculty, sub-department of French at the University of Nairobi. 